\title{
Niedriglohnbeschäftigte auf der Verliererseite
}

\author{
Gerhard Bosch \\ Thorsten Kalina \\ Claudia Weinkopf
}

Der Niedriglohnsektor ist in Deutschland in den vergangenen Jahren deutlich gewachsen. Kontrovers ist jedoch die politische Bewertung dieser Entwicklung: Betreffen Niedriglöhne überwiegend Teilzeitbeschäftigte und Minijobber? Oder erfasst die Zunahme von Niedriglöhnen einen breiteren Kreis? Unsere Analyse zeigt, dass sich die Einkommensverteilung insgesamt zulasten der unteren Einkommensgruppen verschoben hat. Auch Kerngruppen des Arbeitsmarktes sind von Niedriglöhnen betroffen und der wachsende Niedriglohnsektor hat Sogwirkungen in anderen Bereichen entfaltet. Niedriglohnbeschäftigte waren im vergangenen Jahrzehnt auf der Verliererseite: Ihre Löhne sind unterdurchschnittlich gestiegen bzw. inflationsbereinigt sogar gesunken.

\section{Einleitung}

Im internationalen Vergleich galt Deutschland lange als ein Land mit einer geringen Lohnspreizung und nur wenigen niedrig bezahlten Beschäftigten. Noch vor wenigen Jahren wurde intensiv darüber diskutiert, ob man einen Niedriglohnsektor einführen müsse, um Beschäftigung zu fördern. Dabei hat es einen solchen Sektor immer gegeben. Allerdings blieb er lange Zeit stabil bei etwa $13 \%$ bis $14 \%$ der Beschäftigten. Seit Mitte der 1990er Jahre ist der Niedriglohnsektor zunächst fast unbemerkt deutlich gewachsen, ohne dass sich dadurch die Beschäftigungssituation insgesamt oder die Beschäftigungschancen der gering Qualifizierten verbessert hätten. Die Hartz-Reformen haben einen weiteren politisch gewollten Wachstumsschub der Niedriglohnbeschäftigung ausgelöst, dessen Ende nicht absehbar ist. Mittlerweile haben mehrere Studien diese Entwicklung nachgezeichnet. Wir wissen jetzt, dass die Quote der Niedriglohnbeschäftigten in Deutschland gegen den Trend in den meisten anderen EULändern stark gewachsen ist und inzwischen fast den Anteil in den USA erreicht hat (Abbildung 1) (Solow 2007).

Umstritten ist hingegen, ob die wachsende Ungleichheit in der Einkommensverteilung nur ein $\mathrm{zu}$ vernachlässigendes Randphänomen ist oder zunehmend auch den Kern des Beschäftigungssystems trifft. Dies wird kontrovers diskutiert. Die erste These wurde kürzlich vom Deutschen Institut für Wirtschaftsforschung (DIW Berlin) mit dem Hinweis vertreten, dass von Niedriglohnbeschäftigung überwiegend Teilzeitkräfte betroffen seien, die nur einen
Nebenverdienst anstrebten (Brenke/Ziemendorff 2008; DIW 2008). Da viele Haushalte auf das Einkommen aus Teilzeitarbeit angewiesen sind (Statistisches Bundesamt 2004) und eine wachsende Zahl diese geringen Verdienste durch Arbeitslosengeld II aufstocken muss (Bundesagentur für Arbeit 2008), kann man mit guten Gründen eine solche Bewertung infrage stellen. $\mathrm{Zu}-$ dem spricht auch die große Zahl von Vollzeitbeschäftigten mit geringen Löhnen gegen die These einer „Einhegung“ der Niedriglohnbeschäftigung in ein klar abgegrenztes Arbeitsmarktsegment.

Die Zunahme der Niedriglohnbeschäftigung ist in eine umfassendere Umverteilung der Einkommen von unten nach oben eingebettet. Um Gewinner und Verlierer dieser Umverteilung identifizieren zu können, genügt es daher nicht, nur die Entwicklung und Struktur der Niedriglohnbeschäftigung zu analysieren, sondern man

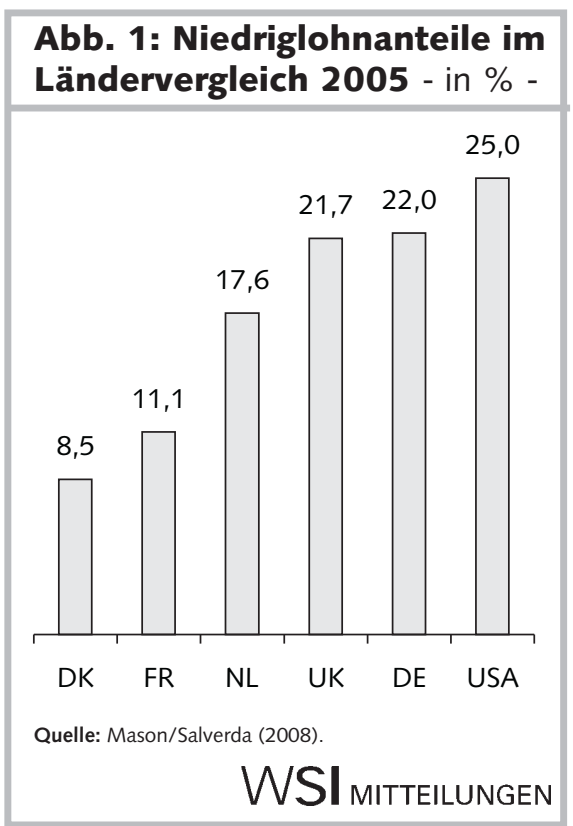

muss auch die Sogwirkungen eines wachsenden Niedriglohnsektors auf die angrenzenden Einkommensgruppen mit in den Blick nehmen. Um ein detailliertes Bild über Dynamik, Betroffenheit und Sogwirkungen des Niedriglohnsektors zeichnen zu können, haben wir Daten des sozio-ökonomischen Panels (SOEP) und des Beschäftigtenpanels der Bundesagentur für Arbeit (BA) ausgewertet. Das SOEP bietet die Möglichkeit, auch Teilzeitbeschäftigte und Minijobs einzubeziehen, derzeit bis zum Jahr 2006. Das BA-Beschäftigtenpanel kann wegen fehlender Angaben zur Arbeitszeit nur für die Löhne von Vollzeitbeschäftigten ausgewertet werden, hat aber wegen seiner erheblich größeren Fallzahlen

Gerhard Bosch, Prof. Dr., Geschäftsführender Direktor des Instituts Arbeit und Qualifikation (IAQ) und Professor für Soziologie an der Universität Duisburg-Essen. Arbeitsschwerpunkte: Arbeitsmarkt und Beschäftigung, Berufliche Bildung, Industrielle Beziehungen.

e-mail: gerhard.bosch@uni-due.de Thorsten Kalina, Diplom-Sozialwissenschaftler, Wissenschaftlicher Mitarbeiter in der Forschungsabteilung "Flexibilität und Sicherheit" des IAQ. Arbeitsschwerpunkte: Arbeitsmarkt und Beschäftigung, Niedriglöhne.

e-mail: thorsten.kalina@uni-due.de Claudia Weinkopf, Dr., Stellvertretende Geschäftsführende Direktorin des IAQ und Leiterin der Forschungsabteilung "Flexibilität und Sicherheit". Arbeitsschwerpunkte: Arbeitsmarkt und Beschäftigung, Niedriglöhne.

e-mail: claudia.weinkopf@uni-due.de 
den Vorteil, die Entwicklung in einzelnen Branchen differenzierter zu analysieren.

Wir untersuchen im Folgenden zunächst die Veränderung der Einkommensverteilung insgesamt (Abschnitt 2). In Abschnitt 3 zeichnen wir ein differenziertes Bild des Niedriglohnsektors: Wie hat er sich entwickelt? Wer ist besonders betroffen? In Abschnitt 4 steht im Mittelpunkt, wie sich Niedrig- und Durchschnittslöhne nach Betriebsmerkmalen entwickelt haben. In Abschnitt 5 gehen wir auf die Ursachen und Triebkräfte der wachsenden Niedriglohnbeschäftigung und den politischen Handlungsbedarf ein. ${ }^{1}$

\section{Die Umverteilung der Einkommen seit 1995}

Die Schere zwischen hohen und niedrigen Einkommen geht in Deutschland seit 1995 immer weiter auf. Ein einfacher Vergleich der inflationsbereinigten Einkommensverteilung in den Jahren 1995, 2000 und 2006 zeigt eine deutliche Abflachung der Kurve mit starken Anteilsverlusten in der Mitte und einer Zunahme am unteren und oberen Ende (Abbildung 2). Auffällig ist die starke Zunahme der Beschäftigung im untersten Lohnbereich (Bruttostundenlöhne unter $6 €$ in Preisen von 1995 und unter rund $7 €$ in Preisen von 2006). ${ }^{2}$

Folge dieser Verschiebung in der Einkommensverteilung ist eine deutliche Zunahme der Einkommensungleichheit. Durch die starke Zunahme gering bezahlter Arbeit auf der einen und die (moderatere) Zunahme gut bezahlter Arbeit auf der anderen Seite hat sich vor allem die Relation des untersten Dezils der Einkommensverteilung zum mittleren Dezil von 1,79 auf 2,18 deutlich verschlechtert, während der Abstand des mittleren zum oberen Dezil weitgehend unverändert blieb ( $\mathrm{Ta}$ belle 1$){ }^{3}$

\begin{tabular}{|c|c|c|c|}
\hline \multicolumn{4}{|c|}{$\begin{array}{l}\text { Tabelle 1: Entwicklung der } \\
\text { Einkommensungleichheit* }\end{array}$} \\
\hline & 1995 & 2000 & 2006 \\
\hline D9/D5 & 1,75 & 1,74 & 1,77 \\
\hline D9/D1 & 3,13 & 3,23 & 3,86 \\
\hline D5/D1 & 1,79 & 1,85 & 2,18 \\
\hline \multicolumn{4}{|c|}{$\begin{array}{l}\text { *Stundenlöhne, alle abhängig Beschäftigten. } \\
\text { Quelle: SOEP 2006; Auswertung der Autoren. }\end{array}$} \\
\hline
\end{tabular}

\section{Abb. 2: Verteilung der Stundenlöhne in Gesamtdeutschland}

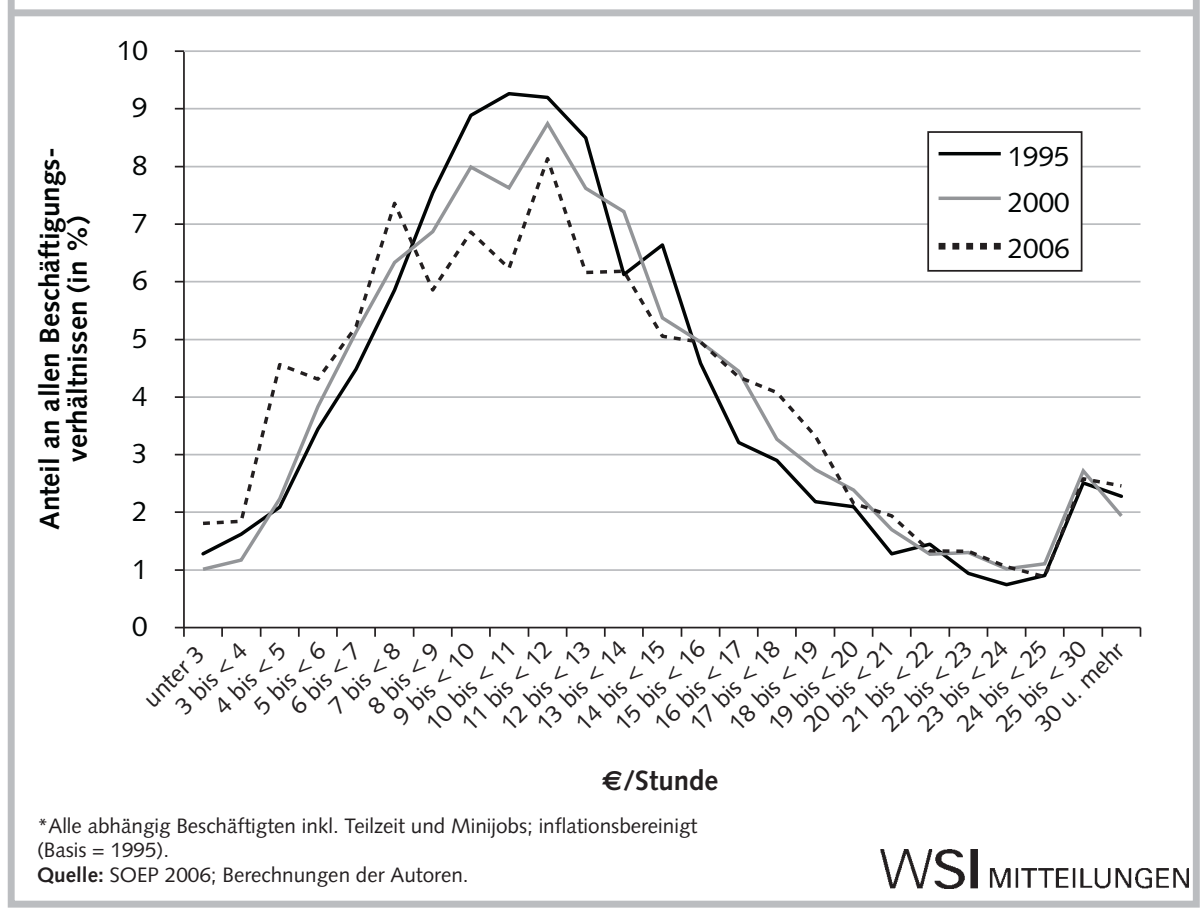

Tabelle 2: Anteile der niedrigen, mittleren und hohen Stundenlöhne* - in \% -

\begin{tabular}{lccc|}
\hline Stundenlohn & $\mathbf{1 9 9 5}$ & $\mathbf{2 0 0 0}$ & $\mathbf{2 0 0 6}$ \\
\hline Niedrig (weniger als 2/3 des Medians) & 15,0 & 17,5 & 22,2 \\
Mittel (2/3 bis 4/3 des Medians) & 63,2 & 59,0 & 51,6 \\
Hoch (mehr als 4/3 des Medians) & 21,8 & 23,6 & 26,3 \\
\hline *Alle abhängig Beschäftigten, getrennte Medianberechnung für Ost und West-Deutschland. & WVI MITTEILUNGEN \\
Quelle: SOEP 2006; Berechnungen der Autoren. &
\end{tabular}

Durch diese Verschiebung der Einkommensgruppen nach unten und nach oben wurden die mittleren Einkommensgruppen ausgedünnt. Sie machten 1995 noch gut $63 \%$ aller Beschäftigten aus und verloren bis 2006 mehr als 11 Prozentpunkte, die sich in entsprechenden Zuwächsen am oberen und stärker noch am unteren Ende der Einkommensverteilung niederschlugen

1 Unsere entsprechenden Untersuchungen wurden im Rahmen eines großen Forschungsnetzwerkes mit Teams aus Dänemark, Frankreich, Großbritannien und den Niederlanden zwischen 2004 und 2007 durchgeführt. Gefördert wurden die Studien, die auch qualitative Branchenanalysen und Betriebsfallstudien beinhalteten, durch die Russell Sage Foundation. Zu den Ergebnissen für Deutschland vgl. Bosch/Weinkopf 2007, 2008.

2 In der Berechnung sind alle abhängig Beschäftigten (Vollzeit, Teilzeit und Minijobs) enthalten. Es wurde vor allem die Einkommensinformation aus der Haupttätigkeit ausgewertet. Nur für den Fall, dass hierfür keine Angaben vorlagen, wurden Nebentätigkeiten einbezogen. Dadurch werden Nebenjobs, die zusätzlich zu einer Haupttätigkeit ausgeübt werden, nicht berücksichtigt und jeder Beschäftigte ist mit einem Beschäftigungsverhält-
(Tabelle 2). Grabka/Frick (2008) haben eine ähnliche Ausdünnung der Mittelklasse auf der Ebene der Haushaltseinkommen festgestellt. Da die Primäreinkommen eine wesentliche Einkommensquelle der Haushalte sind und in vielen Haushalten unzureichende Verdienste nicht durch höhere Einkommen anderer Haushaltsmitglieder kompensiert werden können, dürfte die

nis in der Untersuchung enthalten. Ausgeschlossen wurden bestimmte Kategorien von Beschäftigten, für die sich keine sinnvollen Stundenlöhne berechnen lassen oder für die spezielle Entlohnungsregelungen gelten: Selbstständige und Freiberufler, Auszubildende, Praktikanten, Personen in Umschulung/Rehabilitation oder ABM/SAM, Beschäftigte in Behindertenwerkstätten, mithelfende Familienangehörige, Personen in Altersteilzeit, Nicht-Erwerbstätige, Arbeitslose sowie Wehrund Zivildienstleistende. Studenten, Schüler und Rentner wurden ausgeschlossen, da sie in der Regel nur einer Nebentätigkeit nachgehen.

3 Zu ähnlichen Ergebnissen kommen Giesecke/Verwiebe (2008) in einer nach Ost- und Westdeutschland sowie nach Männern und Frauen differenzierten Auswertung. 


\begin{tabular}{|c|c|c|c|c|c|c|c|}
\hline \multirow[t]{2}{*}{ Quartile } & \multicolumn{3}{|c|}{$\begin{array}{l}\text { Durchschnittlicher } \\
\text { Stundenlohn - in Euro - } \\
\text { (nominal) }\end{array}$} & \multicolumn{2}{|c|}{$\begin{array}{c}\text { Veränderung der } \\
\text { Stundenlöhne - in \% - } \\
\text { (nominal) }\end{array}$} & \multicolumn{2}{|c|}{$\begin{array}{c}\text { Reallöhne } \\
\text { - in Euro - } \\
\text { (in Preisen von 1995) }\end{array}$} \\
\hline & 1995 & 2000 & 2006 & 1995-2000 & 2000-2006 & $\begin{array}{l}\text { Reallohn } \\
2006\end{array}$ & $\begin{array}{c}\text { Veränderung } \\
1995-2006 \\
\text { - in \% - }\end{array}$ \\
\hline 1. Quartil & 6,84 & 7,23 & 6,88 & 5,8 & $-4,8$ & 5,90 & $-13,7$ \\
\hline 2. Quartil & 10,39 & 11,15 & 11,73 & 7,3 & $+5,2$ & 10,06 & $-3,2$ \\
\hline 3. Quartil & 13,11 & 14,49 & 16,02 & 10,5 & $+10,6$ & 13,74 & $+4,8$ \\
\hline 4. Quartil & 21,20 & 22,79 & 25,58 & 7,5 & $+12,3$ & 21,94 & $+3,5$ \\
\hline Gesamt & 12,88 & 13,91 & 15,05 & 8,0 & $+8,2$ & 12,90 & $+0,2$ \\
\hline
\end{tabular}

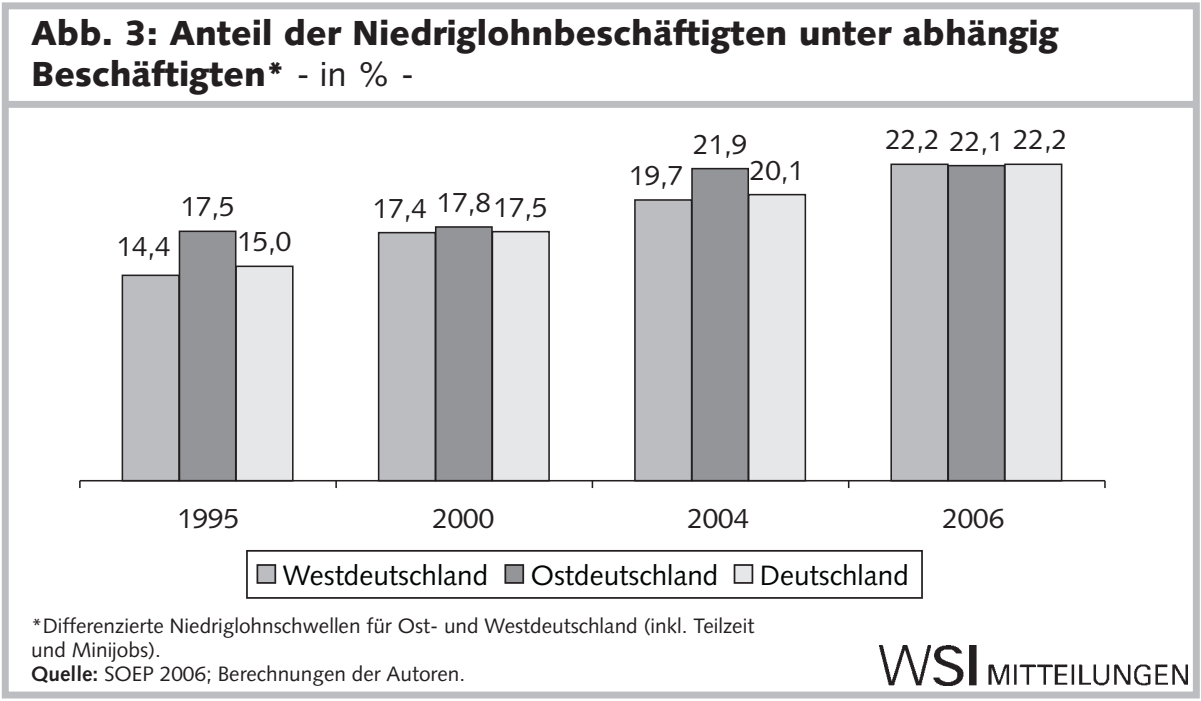

Ausdünnung der mittleren Einkommensgruppen einen hohen Anteil an dieser Entwicklung haben.

Zwischen 1995 und 2006 sind die durchschnittlichen Realeinkommen in Deutschland wegen der nur geringen allgemeinen Lohnsteigerungen kaum gestiegen. Wenn der zu verteilende Kuchen nur wenig wächst und dabei immer ungleicher verteilt wird, ist es wahrscheinlich, dass ein Teil der Beschäftigten Einkommen real und vielleicht sogar nominal verliert. Genau dies können wir seit 1995 beobachten. Im untersten Quartil sind die Reallöhne seit 1995 um fast 14 \% zurückgegangen. ${ }^{4}$ Selbst im zweiten Quartil nahmen sie noch wenn auch weniger deutlich - ab, während sie in den beiden oberen Quartilen anstiegen (Tabelle 3). ${ }^{5}$ Zwischen 2000 und 2006 sanken im unteren Quartil sogar die Nominallöhne; absolut fielen die Löhne unter das Niveau von 2000. Die Einkommensumverteilung hat also die untersten Lohngruppen am härtesten getroffen, Reallohnverluste reichen aber bis in die mittleren Einkommensgruppen hinein. gung entwickelte, deren Wachstum einen wesentlichen Anteil an der Umverteilung der Einkommen hat, und welche Beschäftigungsformen und Personengruppen besonders betroffen sind. Als Niedriglohnschwelle verwenden wir den OECD-Standard von zwei Drittel des Medianlohns. Für Deutschland stellt sich bezogen auf dieses Maß die Frage, ob aufgrund der nach wie vor erheblichen Lohnunterschiede zwischen Ost- und Westdeutschland (Berlemann/Thum 2006) mit zwei getrennten oder einer einheitlichen Niedriglohnschwelle gearbeitet wird. Wie wir an anderer Stelle (Kalina/Weinkopf 2008) ausführlicher gezeigt haben, beeinflusst die Wahl der Vorgehensweise die Ergebnisse zur Gesamtzahl der Beschäftigten im Niedrig- ergeben sich allerdings bezogen auf das Ausmaß der Niedriglohnbeschäftigung in Ostdeutschland: Unter Verwendung differenzierter Schwellen ist hier gut jeder Fünfte von Niedriglöhnen betroffen, während bei einer einheitlichen Niedriglohnschwelle 2006 41,1 \% der ostdeutschen Beschäftigten für Niedriglöhne arbeiteten (Westdeutschland 19,1\%). Die folgenden Auswertungen beruhen auf der Verwendung getrennter Niedriglohnschwellen für Ostund Westdeutschland, damit die Strukturanalysen nicht durch die Einbeziehung eines großen Teils des ostdeutschen Arbeitsmarktes verzerrt werden.

Abbildung 3 gibt einen detaillierteren, auch nach Ost- und Westdeutschland differenzierten Überblick über die Entwicklung des Anteils der Niedriglohnbeschäftigten an allen abhängig Beschäftigten seit 1995. Die Steigerung der gesamtdeutschen Niedriglohnquote von $15 \%$ auf $22,2 \%$ entspricht einem Zuwachs von knapp $48 \%$ innerhalb von nur elf Jahren. In Westdeutschland ist der Niedriglohnanteil sogar um rund $54 \%$ gestiegen (gegenüber $26 \%$ in Ostdeutschland). Die Anzahl der Niedriglohnbeschäftigten in Deutschland ist von rund 4,5 Mio. (1995) auf rund 6,5 Mio. (2006) und damit um 2 Mio. oder gut $43 \%$ gewachsen. ${ }^{6}$ Bemerkenswert ist, dass die Zahl der Niedriglohnbeschäftigten allein zwischen 2004 und 2006 um rund 700.000 gestiegen ist. Es ist davon auszugehen, dass der Zuwachs von Niedriglöhnen durch die Hartz-Reformen im Jahr 2003 beschleunigt worden ist.

$\mathrm{Da}$ es keine allgemeinverbindliche Lohnuntergrenze in Deutschland gibt, ist überdies die Streuung der Löhne nach unten besonders ausgeprägt. Rund 1,9 Mio. lohnsektor kaum. Deutliche Unterschiede
$4 \quad$ Zur Ermittlung der Reallohnentwicklung haben wir den Verbraucherpreisindex aus der Online-Datenbank Genesis des Statistischen Bundesamtes verwendet. Hiernach lag die Preissteigerung zwischen 1995 und 2006 bei $16,6 \%$.

5 Die Einkommen der Vollzeitbeschäftigten haben sich in die gleiche Richtung entwickelt, wenngleich nicht ganz so ausgeprägt. Nach unseren Berechnungen auf der Grundlage des BA-Beschäftigtenpanels musste das unterste Quartil Reallohneinbußen in Höhe von 2,1 \% in Kauf nehmen und das zweite Quartil konnte nur geringe (mit 2,9\% deutlich unter dem Durchschnitt von $8,7 \%$ liegende) Reallohnzuwächse verzeichnen.

6 Zur Abgrenzung der Grundgesamtheit vgl. Fußnote 2. Bezieht man Schüler, Studierende und Rentner ein, lag die Niedriglohnquote 2006 bei 23,5\% und die Zahl der Niedriglohnbeschäftigten insgesamt bei 7,3 Mio. 
Beschäftigte in Deutschland arbeiteten 2006 für Stundenlöhne unter $5 €$ und damit für deutlich weniger (absolut und relativ zum jeweiligen Median) als in Frankreich oder Großbritannien, wo es gesetzliche Mindestlöhne von rund $8 €$ gibt (Caroli/Gautié 2008; Lloyd et al. 2008).

Bemerkenswert ist, dass wegen der Nominallohnverluste im unteren Einkommensquartil zwischen 2004 und 2006 erstmals die Niedriglohnschwellen gesunken sind - von 9,71 € auf 9,61 € in West- und von $7,14 €$ auf $6,81 €$ in Ostdeutschland. Parallel hierzu haben sich auch die durchschnittlichen Stundenlöhne im Niedriglohnsektor sogar nominal verringert um $0,36 €$ in West- und um $0,62 €$ in Ostdeutschland (Tabelle 4). Die Betrachtung der Entwicklung über den Gesamtzeitraum zeigt überdies, dass die Durchschnittslöhne im Niedriglohnsektor inflationsbereinigt 2006 niedriger lagen als 1995 - insbesondere in Ostdeutschland, wo ein Rückgang um $10 \%$ festzustellen ist.

Sind von niedrigen Stundenlöhnen vor allem Teilzeitbeschäftigte und Minijobs betroffen? Zutreffend ist, dass die Anteile dieser Gruppen an allen Niedriglohnbeschäftigten in den letzten Jahren zugenommen haben: Während 1995 Vollzeitbeschäftigte mit einem Anteil von knapp $58 \%$ noch die deutliche Mehrheit der Niedriglohnbeschäftigten stellten, ist ihr Anteil bis 2006 auf gut $46 \%$ gesunken. Entsprechend ist der Anteil von Teilzeitbeschäftigten und Minijobbern auf knapp $54 \%$ der Niedriglohnbeschäftigten gestiegen (Tabelle 5), obwohl ihr Anteil an allen Beschäftigten im SOEP nur 29,5\% beträgt. Besonders stark hat der Anteil der Minijobs am Niedriglohnsektor zugenommen. Er hat sich zwischen 1995 und 2006 nahezu verdoppelt, was vor allem auf das zunehmende beschäftigungspolitische Gewicht der Minijobs zurückzuführen ist: Der Anteil der Minijobs an der Gesamtbeschäftigung hat sich von 2,6\% (1995) auf 7,1 \% (2006) sogar fast verdreifacht. ${ }^{7}$ Dies ist einerseits durch die wachsende Zahl der Minijobs und andererseits durch den deutlichen Rückgang der Gesamtzahl der Vollzeitbeschäftigten bedingt.

Der Rückgang des Anteils von Vollzeitbeschäftigten im Niedriglohnsektor bedeutet allerdings nicht, dass diese 2006 in geringerem Maß als 1995 von Niedriglöhnen betroffen waren. Im Gegenteil: Arbeitete 1995 noch jeder neunte Vollzeitbeschäftigte für einen Niedriglohn, so war es 2006 bereits jeder siebte (Tabelle 6).

\begin{tabular}{|c|c|c|c|c|c|}
\hline & 1995 & 2000 & 2004 & 2006 & $\begin{array}{c}\text { Veränderung } \\
1995 / 2006 \\
\text { in \% }\end{array}$ \\
\hline $\begin{array}{l}\text { Durchschnittlicher Niedriglohn West } \\
\text { (nominal) }\end{array}$ & 5,93 & 6,75 & 7,25 & 6,89 & $+16,2$ \\
\hline $\begin{array}{l}\text { Durchschnittlicher Niedriglohn West } \\
\text { (real, Basis=1995) }\end{array}$ & 5,93 & 6,34 & 6,41 & 5,91 & 0,0 \\
\hline $\begin{array}{l}\text { Durchschnittlicher Niedriglohn Ost } \\
\text { (nominal) }\end{array}$ & 4,63 & 4,95 & 5,48 & 4,86 & $+5,0$ \\
\hline $\begin{array}{l}\text { Durchschnittlicher Niedriglohn Ost } \\
\text { (real, Basis=1995) }\end{array}$ & 4,63 & 4,65 & 4,85 & 4,19 & $-10,0$ \\
\hline
\end{tabular}

\begin{tabular}{|c|c|c|c|c|}
\hline & \multicolumn{2}{|c|}{$\begin{array}{c}\text { Anteil am } \\
\text { Niedriglohnsektor }\end{array}$} & \multicolumn{2}{|c|}{$\begin{array}{c}\text { Anteil an der } \\
\text { Gesamtbeschäftigung }\end{array}$} \\
\hline & 1995 & 2006 & 1995 & 2006 \\
\hline Vollzeit & 57,9 & 46,2 & 79,0 & 70,6 \\
\hline Teilzeit & 27,2 & 24,0 & 18,4 & 22,4 \\
\hline $\begin{array}{l}\text { Geringfügige Beschäftigung } \\
\text { (Minijobs) }\end{array}$ & 14,9 & 29,7 & 2,6 & 7,1 \\
\hline Gesamt & 100,0 & 100,0 & 100,0 & 100,0 \\
\hline \multicolumn{3}{|c|}{ Quelle: SOEP 2006; Berechnungen der Autoren. } & \multicolumn{2}{|c|}{ WSI mitTEILUNG } \\
\hline
\end{tabular}

\begin{tabular}{|c|c|c|c|c|}
\hline \multicolumn{5}{|c|}{$\begin{array}{l}\text { Tabelle 6: Niedriglohnbeschäftigung nach Beschäftigungsformen } \\
\text { - in \% - }\end{array}$} \\
\hline & 1995 & 2006 & Niedriglohnbeschäftigung & Gesamtbeschäftigung \\
\hline Vollzeit & 11,0 & 14,3 & $+12,6$ & $-13,5$ \\
\hline Teilzeit & 22,2 & 23,4 & $+24,5$ & $+18,0$ \\
\hline Minijobs & 86,0 & 91,7 & $+181,2$ & $+163,8$ \\
\hline Gesamt & 15,0 & 22,2 & $+43,3$ & $-3,1$ \\
\hline
\end{tabular}

Noch höher als in unseren Berechnungen auf der Basis des SOEP liegt der Anteil von Vollzeitbeschäftigten, die von Niedriglöhnen betroffen sind, nach Ergebnissen von Analysen mit BA-Daten, die sich auf Vollzeitbeschäftigte beschränken. Für 2004 beziffern Rhein/Stamm (2006, S. 11) die Niedriglohnquote von Vollzeitbeschäftigten auf 18,4 \%; nach unseren eigenen Berechnungen lag dieser Anteil 2005 mit 18,7 \% noch etwas höher. Die unterschiedlichen Quoten von Niedriglöhnen unter Vollzeitbeschäftigten resultieren vor allem daraus, dass die Niedriglohnschwelle in der Gruppe der Vollzeitbeschäftigten höher liegt als unter allen Beschäftigten. Übereinstimmend zeigen vorliegende Studien, dass auch die Gruppe der Vollzeitbeschäftigten seit Mitte der 1990er Jahre zunehmend von Niedriglöhnen betroffen ist (Rhein/Stamm 2006; Bosch/Kalina 2007). Dies sind deutliche Indizien, dass sich Niedriglohnbe- schäftigung in Deutschland nicht alleine oder vorrangig auf Teilzeitbeschäftigte konzentriert.

Ein Ausbau des Niedriglohnsektors wird vielfach für notwendig gehalten, um gering Qualifizierten, die andernfalls vom Arbeitsmarkt ausgeschlossen blieben, überhaupt Beschäftigungschancen zu eröffnen. Tatsächlich zeigen unsere Auswertungen, dass gering Qualifizierte von Niedriglöhnen überproportional und in steigendem Maße betroffen sind: Der Niedriglohnteil dieser Beschäftigtengruppe stieg zwischen 1995 und 2006 um rund $47 \%$. Gleichzeitig lag die Steigerungsrate aber in der zahlenmäßig weitaus größeren Gruppe der Be-

\footnotetext{
7 Diese Angabe bezieht sich auf unsere Grundgesamtheit, in der weder Schüler, Studierende und Rentner noch Nebenjobs enthalten sind. Die Quote in der Gesamtwirtschaft liegt erheblich höher.
} 


\begin{tabular}{|c|c|c|c|c|}
\hline & Kategorie & 1995 & 2000 & 2006 \\
\hline \multirow[t]{3}{*}{ Qualifikation } & Ohne Berufsausbildung & 33,5 & 26,1 & 26,4 \\
\hline & Mit Berufsausbildung & 58,6 & 66,4 & 67,5 \\
\hline & Universität/Fachhochschule & 7,9 & 7,5 & 6,1 \\
\hline \multirow[t]{2}{*}{ Geschlecht } & Männer & 27,5 & 30,6 & 31,9 \\
\hline & Frauen & 72,5 & 69,4 & 68,1 \\
\hline \multirow[t]{5}{*}{ Alter } & unter 25 & 13,8 & 15,2 & 12,3 \\
\hline & $25-34$ & 26,2 & 24,3 & 23,4 \\
\hline & $35-44$ & 22,3 & 25,8 & 29,9 \\
\hline & $45-54$ & 20,8 & 21,0 & 19,8 \\
\hline & $55+$ & 16,9 & 13,7 & 14,7 \\
\hline \multirow[t]{2}{*}{ Nationalität } & Deutsche & 88,5 & 87,8 & 87,9 \\
\hline & Ausländer & 11,5 & 12,2 & 12,1 \\
\hline Gesamt (Deutschland) & & 100,0 & 100,0 & 100,0 \\
\hline
\end{tabular}

schäftigten mit abgeschlossener Berufsausbildung mit fast $70 \%$ noch deutlich höher.

Ein Blick auf die Struktur der Niedriglohnbeschäftigten nach Beschäftigtenmerkmalen (Tabelle 7) macht zudem deutlich, dass 2006 nur noch knapp ein Viertel aller Niedriglohnbeschäftigten aus der Gruppe der formal gering Qualifizierten stammte. Der Anteil von Beschäftigten mit abgeschlossener Berufsausbildung oder einem akademischen Abschluss an der Gesamtzahl der Niedriglohnbeschäftigten ist demgegenüber von $66,5 \%$ (1995) auf 73,6 \% (2006) deutlich gestiegen. Mittlerweile sind also fast drei von vier Niedriglohnbeschäftigten in Deutschland formal qualifizierte Beschäftigte - ein auch im internationalen Vergleich extrem hoher Wert. So haben etwa in den USA rund $70 \%$ der Niedriglohnbeschäftigten keinen oder nur einen High School-Abschluss, also ein Bildungsniveau unterhalb einer abgeschlossenen beruflichen Ausbildung in
Deutschland (CBO 2006, S. 18; Appelbaum et al. 2003). Dieser markante Unterschied im Qualifikationsprofil der Niedriglohnbeschäftigten dürfte zum einen der anhaltend hohen Arbeitslosigkeit in Deutschland geschuldet sein, die den Druck, eine gering bezahlte Tätigkeit anzunehmen, auch für gut Qualifizierte erhöht hat. Zum anderen ist die Gruppe der gering Qualifizierten in Deutschland durch das ausgebaute System der beruflichen Bildung im internationalen Vergleich relativ klein.

\section{Differenzierte Analyse nach Betriebsmerkmalen}

In der Vergangenheit folgte die Lohnentwicklung der kleineren Betriebe und der gewerkschaftlich schwächer organisierten Branchen meistens der Lohnentwicklung in größeren Betrieben und gewerkschaft-

\begin{tabular}{|c|c|c|c|c|c|c|}
\hline \multirow{3}{*}{$\begin{array}{l}\text { Betriebe nach } \\
\text { Beschäftigtenzahl }\end{array}$} & \multicolumn{3}{|c|}{ Niedriglohnanteil in \% } & \multirow{2}{*}{\multicolumn{2}{|c|}{$\begin{array}{l}\text { Durchschnittliches Brutto- } \\
\text { Monatseinkommen, } \\
\text { inflationsbereinigt (in Euro) }\end{array}$}} & \multirow{3}{*}{$\begin{array}{c}\text { Änderung } \\
\begin{array}{c}1998-2005 \\
\text { in \% }\end{array}\end{array}$} \\
\hline & \multirow[t]{2}{*}{1998} & \multirow[t]{2}{*}{2005} & \multirow[t]{2}{*}{$\begin{array}{l}\text { Änderung } \\
1998-2005\end{array}$} & & & \\
\hline & & & & 1998 & 2005 & \\
\hline $1-4$ & 47,1 & 51,8 & $+10,1$ & $1.591,89$ & $1.595,54$ & $+0,2$ \\
\hline $5-9$ & 29,6 & 36,3 & $+22,4$ & $1.861,49$ & $1.888,76$ & $+1,5$ \\
\hline 10-19 & 20,7 & 26,4 & $+27,6$ & $2.021,14$ & $2.099,46$ & $+3,9$ \\
\hline $20-49$ & 15,9 & 21,2 & $+33,4$ & $2.147,02$ & $2.258,72$ & $+5,2$ \\
\hline $50-99$ & 13,2 & 18,1 & $+36,9$ & $2.251,19$ & $2.379,23$ & $+5,7$ \\
\hline 100-199 & 10,9 & 14,7 & $+34,7$ & $2.340,19$ & $2.514,66$ & $+7,5$ \\
\hline 200-499 & 7,5 & 9,3 & $+23,3$ & $2.457,91$ & $2.725,67$ & $+10,9$ \\
\hline 500 und mehr & 3,1 & 3,3 & $+7,0$ & $2.720,19$ & $3.116,15$ & $+14,6$ \\
\hline Gesamtwirtschaft & 15,5 & 18,7 & $+20,5$ & $2.263,19$ & $2.460,09$ & $+8,7$ \\
\hline
\end{tabular}

lich gut organisierten Branchen. Dieser tarifliche Geleitzug, der für eine gleichmäßige Verteilung des Wohlstandszuwachses über die gesamte Wirtschaft in Deutschland sorgte, hat sich - so unsere These mittlerweile aufgelöst. In gewerkschaftlich schwächer organisierten Branchen lassen sich nicht mehr die gleichen Tariferhöhungen wie im verarbeitenden Gewerbe durchsetzen. Davon ist eine wachsende Anzahl von Beschäftigten betroffen, da die Beschäftigung vor allem in schlecht organisierten Dienstleistungsbranchen und dort vor allem in Klein- und Mittelbetrieben ohne Betriebsräte wächst. Zusätzlich werden Tätigkeiten aus gut organisierten Bereichen in tariffreie Zonen des Arbeitsmarktes ausgelagert, da solches Outsourcing wegen des wachsenden Lohngefälles für die Unternehmen immer attraktiver wird.

Um diese Thesen zu überprüfen, haben wir das BA-Beschäftigtenpanel ausgewertet, das zwar bezogen auf Löhne nur Auswertungen für Vollzeitbeschäftigte zulässt, aber wegen seiner hohen Fallzahlen detailliertere Analysen nach Betriebsgrößenklassen und Wirtschaftszweigen ermöglicht. Im Vergleich zu den Analysen mit dem SOEP ist zunächst bemerkenswert, dass für Vollzeitbeschäftigte keine Reallohnverluste zu verzeichnen sind.

Unsere Analyse zeigt aber, dass die Lohnunterschiede zwischen Groß- und Kleinbetrieben zwischen 1998 und 2005 deutlich zugenommen haben. Während Vollzeitbeschäftigte in Kleinbetrieben 1998 im Durchschnitt noch etwa 59 \% des Lohnniveaus von Großbetrieben erreichten, lag diese Quote 2005 nur noch bei rund $51 \%$. Wie stark sich die Betriebsgrößenklassen bei der Lohnentwicklung auseinander bewegt haben, zeigt sich sehr deutlich beim Vergleich des inflationsbereinigten Einkommensanstiegs. Während sich die durchschnittlichen Monatslöhne in Kleinbetrieben zwischen 1998 und 2005 mit 0,2 \% kaum erhöhten, war der Lohnzuwachs mit zunehmender Betriebsgröße immer größer und lag in Großbetrieben immerhin bei knapp $15 \%$ (Tabelle 8).

Aus Tabelle 8 ist auch erkennbar, dass die Niedriglohnanteile mit wachsender Betriebsgröße abnehmen: In Kleinstbetrieben ( 1 bis 4 Beschäftigte) arbeiteten 2005 mehr als die Hälfte der Vollzeitbeschäftigten für Niedriglöhne, während in Großbetrieben (500 und mehr Beschäftigte) fast unverändert nur rund $3 \%$ der Beschäftigten gering bezahlt wurden. Der Abstand zwischen 
Groß- und Kleinbetrieben ist im Zeitverlauf noch gewachsen.

Um zu untersuchen, ob sich die Lohnentwicklung nach Wirtschaftszweigen ausdifferenziert, haben wir die Wirtschaftszweige nach ihrem Niedriglohnanteil 1998 in drei Gruppen eingeteilt: Erstens Wirtschaftszweige mit einem geringen Niedriglohnanteil (weniger als $50 \%$ des gesamtwirtschaftlichen Wertes), zweitens Wirtschaftszweige mit einem mittleren Niedriglohnanteil (zwischen 50 und $150 \%$ des gesamtwirtschaftlichen Wertes) und drittens Wirtschaftszweige mit einem hohen Niedriglohnanteil (über $150 \%$ des gesamtwirtschaftlichen Wertes) (Tabelle 9).

In der Gruppe der Wirtschaftszweige mit geringen Niedriglohnanteilen stieg der durchschnittliche Niedriglohnanteil von $4,4 \%$ auf rund 5,3\% (2005), was leicht unter der Wachstumsrate insgesamt lag. Wegen des geringen Ausgangswertes entsprach dieser Anstieg jedoch nur 0,9 Prozentpunkten. In einigen Wirtschaftszweigen ist der Niedriglohnanteil sogar gesunken (am deutlichsten im Fahrzeugbau). Der durchschnittliche Bruttomonatslohn lag in dieser Gruppe deutlich über dem gesamtwirtschaftlichen Wert und ist zudem zwischen 1998 und 2005 stärker gestiegen als in der Gesamtwirtschaft.

In der Gruppe mit einem mittleren Niedriglohnanteil zwischen 50 und $150 \%$ des gesamtwirtschaftlichen Wertes war der Anstieg des Niedriglohnanteils bis 2005 prozentual am stärksten $(+26,2 \%)$, in Prozentpunkten gemessen mit $+3,5$ aber moderater. Die realen Bruttomonatseinkommen sind nur um 6,3\% und damit unterdurchschnittlich gestiegen.

In der Gruppe mit hohen Niedriglohnanteilen ist der Zuwachs von Niedriglöhnen prozentual gerechnet (mit Ausnahme der Dienstleistungen für Unternehmen) unterdurchschnittlich gewesen, was mit den ohnehin schon sehr hohen Anteilen von Niedriglöhnen zusammenhängen dürfte. In Prozentpunkten gerechnet war der Anstieg mit 5,1 hingegen deutlich höher als in den beiden anderen Gruppen. Die Lohnentwicklung war insgesamt und in den Teilbranchen ausnahmslos unterdurchschnittlich (im Gastgewerbe sogar rückläufig), was zusammen genommen auf eine zunehmende Entkoppelung der Niedriglohnbranchen von der gesamtwirtschaftlichen Lohnentwicklung hindeutet.

Insgesamt ist festzuhalten, dass der Abstand zwischen den Branchen mit einem

\begin{tabular}{|c|c|c|c|c|c|c|}
\hline \multirow{3}{*}{$\begin{array}{l}\text { Wirtschaftszweige nach } \\
\text { Niedriglohnanteil }\end{array}$} & \multicolumn{3}{|c|}{ Niedriglohnanteil in \% } & \multirow{2}{*}{\multicolumn{2}{|c|}{$\begin{array}{l}\text { Durchschnittliches } \\
\text { Brutto- } \\
\text { Monatseinkommen, } \\
\text { inflationsbereinigt } \\
\text { in Euro }\end{array}$}} & \multirow{3}{*}{$\begin{array}{l}\text { Änderung } \\
1998 / 2005 \\
\text { in \% }\end{array}$} \\
\hline & \multirow[t]{2}{*}{1998} & \multirow[t]{2}{*}{2005} & \multirow[t]{2}{*}{$\begin{array}{l}\text { Änderung } \\
1998 / 2005\end{array}$} & & & \\
\hline & & & & 1998 & 2005 & \\
\hline $\begin{array}{l}\text { Wirtschaftszweige mit } \\
\text { geringem Niedriglohnanteil } \\
\text { (<50\% des Gesamtniveaus) }\end{array}$ & 4,4 & 5,3 & $+18,2$ & $2.605,40$ & $2.933,06$ & $+12,6$ \\
\hline $\begin{array}{l}\text { Wirtschaftszweige mit } \\
\text { mittlerem Niedriglohnanteil } \\
\text { (50-150\% des } \\
\text { Gesamtniveaus) }\end{array}$ & 13,4 & 16,9 & $+26,2$ & $2.223,16$ & $2.363,04$ & $+6,3$ \\
\hline $\begin{array}{l}\text { Wirtschaftszweige mit } \\
\text { hohem Niedriglohnanteil } \\
\text { (>150\% des Gesamtniveaus) }\end{array}$ & 36,5 & 41,6 & $+14,1$ & $1.828,78$ & $1.944,63$ & $+6,3$ \\
\hline Gesamtwirtschaft & 15,5 & 18,7 & $+20,5$ & $2.263,19$ & $2.460,09$ & $+8,7$ \\
\hline
\end{tabular}

geringen Niedriglohnanteil und denen mit mittlerem und hohem Niedriglohnanteil gewachsen ist. Gleichzeitig sind im oberen Beschäftigungssegment mit unterdurchschnittlichen Niedriglohnanteilen auch die Löhne deutlich stärker gestiegen als in den beiden anderen Beschäftigungssegmenten. ${ }^{8}$

\section{2 \\ Ursachen und politischer Handlungsbedarf}

Unsere Analyse hat gezeigt, dass sich die Löhne in Deutschland seit Mitte der 1990er Jahre erheblich auseinanderentwickelt haben und der Niedriglohnsektor stark gewachsen ist. Deutschland hatte damit bereits 2005 den höchsten Niedriglohnanteil in der EU und liegt nicht mehr weit von den USA entfernt. Der Anteil der gering Bezahlten ist sowohl bei den Vollzeit- als auch bei den Teilzeitbeschäftigten (einschließlich Minijobs) deutlich gestiegen. Betroffen von Niedriglöhnen sind nicht nur spezielle Personengruppen wie Jüngere oder gering Qualifizierte, sondern zunehmend auch die mittleren Altersgruppen und qualifizierte Beschäftigte. Die Umverteilung der Löhne hat sich nicht auf die unterste Einkommensgruppe beschränkt, sondern reicht bis in die mittleren Einkommensgruppen hinein. Die Mittelschicht in der Einkommensverteilung ist durch Verluste nach unten und Aufstiege nach oben erheblich ausgedünnt worden. Gleichzeitig haben sich die Lohnunterschiede zwischen Großbetrieben und kleineren Betrieben, die oft keine Betriebsräte haben, sowie zwischen den Branchen mit hoher und geringer Tarifbindung deutlich erhöht.

Wie konnte es zu einer so raschen $\mathrm{Zu}$ nahme von Niedrig- und sogar Niedrigstlöhnen in einer sozialen Marktwirtschaft wie Deutschland kommen? Den wichtigsten Grund sehen wir in der Anfälligkeit des deutschen Tarifsystems für Außenseiterkonkurrenz. Da es keine verbindlichen Lohnuntergrenzen - durch einen gesetzlichen Mindestlohn oder allgemeinverbindliche Tarifverträge - gibt, ist es möglich, auch Löhne unterhalb der Branchentarife zu zahlen. Bis zur deutschen Wiedervereinigung spielte dies allerdings nur eine untergeordnete Rolle. Rund 80 \% der Unternehmen waren tarifgebunden und die restlichen Unternehmen orientierten sich an diesen Größen, da anderenfalls ihre Arbeitskräfte abgewandert wären. Diese Ängste schwanden jedoch mit der hohen Arbeitslosigkeit nach der Wiedervereinigung. Zunächst in Ostdeutschland und dann auch Westdeutschland verließen viele Unternehmen die Arbeitgeberverbände oder traten erst gar nicht ein, um geringere Löhne zahlen zu können.

Der ab Mitte der 1990er Jahre wachsende Niedriglohnsektor hat Sogwirkungen selbst für gewerkschaftlich gut organisierte Unternehmen entfaltet. Das wachsende Lohngefälle in der Wirtschaft bietet zunehmend Anreize, zuvor gut bezahlte Tätigkeiten in Branchen und Betriebe mit geringeren Löhnen zu verlagern. Diese Entwicklung ist durch politische Interventio-

8 Weiterführende Daten und Auswertungen sind be den Autoren erhältlich. 
nen massiv gefördert worden. Starken Antrieb erhielt der Außenseiterwettbewerb durch veränderte europäische Wettbewerbsregelungen. Mit der Dienstleistungsfreiheit können Arbeitskräfte nach Deutschland zu Lohnbedingungen ihres Heimatlandes entsendet werden. Nur in der Bauwirtschaft wurden tariflich vereinbarte Mindestlöhne nach dem Entsendegesetz für allgemeinverbindlich erklärt. In anderen Branchen wie etwa der Fleischindustrie sind demgegenüber ohnehin schon gering verdienende heimische Arbeitskräfte durch noch schlechter bezahlte Werkvertragsnehmer ersetzt worden (Czommer 2007). Noch weitreichendere Auswirkungen hatten die EU-Direktiven zur Öffnung der Produktmärkte ehemals staatlicher Dienstleistungen (z. B. im Bereich Post, Telekommunikation oder Nahverkehr). Während sich neue Anbieter solcher Dienstleistungen in den meisten anderen europäischen Ländern an allgemein verbindliche Tarifverträge halten müssen, können sie in Deutschland jeden auf dem Markt durchsetzbaren Lohn zahlen. Dies lädt Unternehmen zu Geschäftsmodellen ein, die nicht mehr auf Innovationen, sondern auf Lohndrückerei basieren, wie es z. B. im Bereich der Postdienstleistungen zu beobachten war.

Einen weiteren politischen Schub gab es durch die Agenda 2010 und die HartzReformen. Durch die Abschaffung der einkommensabhängigen Arbeitslosenhilfe ist vor allem der Druck auf qualifizierte Langzeitarbeitslose, die zuvor gut verdient hatten, erhöht worden, auch einen schlecht bezahlten Arbeitsplatz anzunehmen. Die Aufhebung der zeitlichen Beschränkungen für den Verleih von Arbeitskräften hat Unternehmen die Möglichkeit eröffnet, Stammbeschäftigte auch dauerhaft durch geringer entlohnte Leiharbeitskräfte $\mathrm{zu}$ ersetzen.
Die Vorschrift zur gleichen Bezahlung von Leiharbeitskräften, die solche Praktiken einschränken sollte, ist ins Leere gelaufen, weil Zeitarbeitsunternehmen von diesem Prinzip durch die Anwendung eines Tarifvertrags abweichen können. Inzwischen sind zahlreiche Tarifverträge für die Zeitarbeit abgeschlossen worden, die vor allem in den Einstiegsgruppen Stundenlöhne deutlich unterhalb der Niedriglohnschwelle vorsehen und auch insgesamt im Vergleich zu den Tariflöhnen in typischen Einsatzbranchen erheblich niedriger liegen (Weinkopf 2006). Schließlich ist noch die deutliche Heraufsetzung der Verdienstgrenze für Minijobs zu nennen. Für Beschäftigte in Minijobs gelten zwar im Prinzip alle arbeitsrechtlichen Schutzvorschriften und sie haben Anspruch auf denselben Stundenlohn wie vergleichbare sozialversicherungspflichtig Beschäftigte. In der Praxis ist aber ein besonderer Beschäftigtenstatus geschaffen worden, dem diese Rechte häufig vorenthalten werden.

Die politisch gewollte Ausweitung des Niedriglohnsektors hat auch dazu beigetragen, dass die durchschnittlichen Stundenlöhne im unteren Einkommensquartil inflationsbereinigt im Vergleich zu 1995 um fast $14 \%$ gesunken sind. Der Anteil der Niedriglohnbeschäftigten ist in Deutschland selbst im Wirtschaftsaufschwung seit 2004 weiter angestiegen, und die durchschnittlichen Stundenlöhne im Niedriglohnsektor sind zuletzt sogar nominal rückläufig gewesen. Dass Niedriglohnbeschäftigte im letzten Jahrzehnt im Vergleich $\mathrm{zu}$ anderen Beschäftigtengruppen deutlich verloren haben, lässt sich auch daran festmachen, dass die durchschnittlichen Stundenlöhne im Niedriglohnsektor im Vergleich von 1995 und 2006 inflationsbereinigt nicht gestiegen und in Ostdeutschland sogar gesunken sind.

Um diese Entwicklung zu stoppen und ein weiteres Anwachsen des Niedriglohnsektors zu verhindern, muss politisch umgesteuert werden. In einer Wirtschaft mit offenen Arbeitsmärkten und deregulierten Produktmärkten ist der Weg zurück in die „guten alten Zeiten“ vor 1990 verbaut. Man muss neue Wege finden, um verbindliche Lohnuntergrenzen auch für Bereiche festzulegen, in denen die Gewerkschaften und Arbeitgeberverbände nicht präsent oder zu schwach sind. Um Niedrigstlöhne und Lohndumping wirksam zu unterbinden, halten wir die Einführung eines allgemeinen gesetzlichen Mindestlohnes für erforderlich, der - wie das britische Erfolgsbeispiel zeigt - bei angemessener Höhe auch der Beschäftigung nicht schadet (Bosch 2007; Bosch/Weinkopf 2006). Allein auf branchenbezogene Lösungen zu setzen, erscheint nicht ausreichend, weil hierbei gerade einige derjenigen Branchen außen vor blieben, in denen Niedriglöhne besonders verbreitet sind. $\mathrm{Zu}$ einem größeren Reformpaket gehören aber auch die Aufhebung des Sonderstatus der Minijobs und ihre Eingliederung in das System der sozialversicherungspflichtigen Beschäftigung sowie die Durchsetzung gleicher Bezahlung von Leiharbeitskräften. Nicht zuletzt muss die Allgemeinverbindlichkeit von Tarifverträgen erleichtert werden. Dies erscheint umso dringlicher, als nach dem Urteil des Europäischen Gerichtshofs von April 2008 weniger umfassende Maßnahmen zur Durchsetzung von Mindeststandards, wie etwa Tariftreuegesetze bei öffentlicher Vergabe, die nicht für alle Unternehmen einer Branche gelten, nicht mehr zulässig sind (Schulten/Pawicki 2008). 
Appelbaum, E./Bernhardt, A./Murnane, R. J. (2003): Low-Wage America: An Overview, in: Dies. (Hrsg.): Low-Wage America. How Employers Are Reshaping Opportunity in the Workplace, New York, S. 1-29 Berlemann, M./Thum, M. (2006): Mittelfristige Perspektiven der OstWest-Konvergenz, in: Ifo Dresden berichtet 1, S. 34-39

Bosch, G. (2007): Mindestlohn in Deutschland notwendig - Kein Gegensatz zwischen sozialer Gerechtigkeit und Beschäftigung, in: Zeitschrift für Arbeitsmarktforschung 4, S. 421-429

Bosch, G./Kalina, T. (2007): Niedriglohnbeschäftigung in Deutschland, in: Bosch, G./Weinkopf, C. (Hrsg.): Arbeiten für wenig Geld: Niedriglohnbeschäftigung in Deutschland, Frankfurt, S. 20-105

Bosch, G./Weinkopf, C. (2006): Mindestlöhne in Großbritannien Ein geglücktes Realexperiment, in: WSI-Mitteilungen 3, S. 125-130 Bosch, G./Weinkopf, C. (Hrsg.) (2007): Arbeiten für wenig Geld: Niedriglohnbeschäftigung in Deutschland, Frankfurt

Bosch, G./Weinkopf, C. (Hrsg.) (2008): Low-Wage Work in Germany, The Russell Sage Foundation Case Studies of Job Quality in Advanced Economies, New York

Bundesagentur für Arbeit (2008): Arbeitsmarkt in Zahlen: Erwerbstätigkeit von erwerbsfähigen Leistungsbeziehern September 2007, Bericht der Statistik der BA, Nürnberg

Brenke, K./Ziemendorff, J. (2008): Hilfebedürftig trotz Arbeit? - kein Massenphänomen in Deutschland, in: DIW-Wochenbericht 4, S. 33-40 Caroli, E./Gautié, J. (Hrsg.) (2008): Low-Wage Work in France. The Russell Sage Foundation Case Studies of Job Quality in Advanced Economies, New York

Congressional Budget Office (CBO) (2006): Changes in the low-wage labor markets between 1979 and 2005, Congress of the United States, Washington

Czommer, L. (2007): Wildwestzustände in Deutschland? Einfacharbeitsplätze in der Ernährungsindustrie, in: Bosch, G./Weinkopf, C. (Hrsg.): Arbeiten für wenig Geld: Niedriglohnbeschäftigung in Deutschland, Frankfurt, S. 142-174
Deutsches Institut für Wirtschaftsforschung (DIW) (2008): Der Niedriglohnsektor in Deutschland wächst nicht mehr, Stellungnahme vom 8. Februar 2008, Berlin

Giesecke, J./Verwiebe, R. (2008): Die Lohnentwicklung in Deutschland zwischen 1998 und 2005 - Wachsende Ungleichheit, in: WSI-Mitteilungen 2, S. 85-91

Grabka, M. M./Frick, J. R. (2008): Schrumpfende Mittelschicht - Anzeichen einer dauerhaften Polarisierung der verfügbaren Einkommen?, in: DIW-Wochenbericht 10, S. 101-108

Kalina, T./Weinkopf, C. (2008): Weitere Zunahme der Niedriglohnbeschäftigung: 2006 bereits rund 6,5 Mio. Beschäftigte betroffen, IAQ-Report 2008-01

Lloyd, C./Mason, G./Mayhew, K. (Hrsg.) (2008): Low-Wage Work in the United Kingdom, The Russell Sage Foundation Case Studies of Job Quality in Advanced Economies, New York

Mason, G./Salverda, W. (2008): Low Pay, Living Standards and Employment, Manuskript im Rahmen der Phase II des Forschungsnetzwerkes “The Russell Sage Foundation Case Studies of Job Quality in Advanced Economies", New York

Rhein, T./Stamm, M. (2006): Niedriglohnbeschäftigung in Deutschland deskriptive Befunde zur Entwicklung seit 1980 und Verteilung auf Berufe und Wirtschaftszweige, IAB-Forschungsbericht 12, Nürnberg Schulten, T./Pawicki, M. (2008): Tariftreueregelungen in Deutschland Ein aktueller Überblick, in: WSI-Mitteilungen 4, S. 184-190

Solow, R. M. (2007): Vorwort, in: Bosch, G./Weinkopf, C. (Hrsg.): Arbeiten für wenig Geld: Niedriglohnbeschäftigung in Deutschland, Frankfurt, S. 7-14

Statistisches Bundesamt (2004): Zwei von drei Teilzeitkräften leben wesentlich vom Arbeitseinkommen, Pressemitteilung vom 14. Oktober, Bonn

Weinkopf, C. (2006): Mindestbedingungen für die Zeitarbeitsbranche? Expertise im Auftrag des Interessenverbandes Deutscher Zeitarbeitsunternehmen (iGZ), Institut Arbeit und Technik, Gelsenkirchen 